Population Study

Oral

Abstract ID: 36

\title{
Noise exposure during orthopaedic surgery
}

\author{
Ailin Razali $^{\mathrm{a}} \mid$ Hendy Putra Herman ${ }^{\mathrm{b}} \mid$ Ahmad Hafiz Zulkifly ${ }^{\mathrm{a}}$ \\ ${ }^{a}$ Kulliyyah of Medicine, International Islamic University Malaysia \\ ${ }^{b}$ Academy of Sciences Malaysia
}

Introduction: There is serious concern on noise hazard in the operating theatre, especially in the orthopaedics field that requires usage of many instruments. However, local data on this noise exposure is rather limited. This study was conducted as a pilot study to investigate the noise exposure in orthopaedics theatre in a hospital in Kuantan, Pahang in June 2011. Methods: Several different orthopaedics theatres involving procedures that use powered instruments were investigated. Time recorded noise exposures were taken using a noise dosimeter throughout the surgeries worn by the surgeons. The peak noise level and the average noise exposure within 8 hours were determined, and compared with available health guidelines, namely the Factories and Machinery (Noise Exposure) Regulations 1989. Results: Six out of seven surgeries which include interlocking nail and plating of femur, Ilizarof insertion, total knee replacement and dynamic hip screw insertion of the femoral head were found to exceed $140 \mathrm{~dB}(\mathrm{~A})$ for peak sound levels. The average noise exposure within 8 hours was below $85 \mathrm{~dB}(\mathrm{~A})$ in all cases. Although the average noise level did not surpass the health guidelines, the peak level exceeding $140 \mathrm{~dB}(\mathrm{~A})$ still carries risk for hearing loss. Conclusions: In conclusion, healthcare personnel in operating theatre were exposed to noise hazard for certain orthopaedics surgical procedures. Hence, proper noise management should be implemented to protect them from noise-induced hearing loss while maintaining the success and efficiency of the surgeries.

KEYWORDS: Noise exposure, orthopaedics, noise-induced hearing loss 